\title{
Mapping Intellectual Structure and Research Performance for the Nanoparticles in Pancreatic Cancer Field
}

This article was published in the following Dove Press journal: International Journal of Nanomedicine

\author{
Xuan Zhu ${ }^{1-3}$ \\ Qingquan Kong ${ }^{2}$ \\ Xing $\mathrm{Niu}^{4}$ \\ Lijie Chen ${ }^{4}$ \\ Chunlin $\mathrm{Ge}\left(\mathbb{D}^{\prime}\right.$ \\ 'Department of General Surgery, The \\ First Affiliated Hospital of China Medical \\ University, Shenyang, Liaoning I I000I, \\ People's Republic of China; ${ }^{2}$ Institute of \\ Translational Medicine, China Medical \\ University, Shenyang, Liaoning II 0122 , \\ People's Republic of China; ${ }^{3}$ Anshan \\ Hospital, The First Affiliated Hospital of \\ China Medical University, Anshan, \\ Liaoning I I 40 I I, People's Republic of \\ China; ${ }^{4}$ Department of Second Clinical \\ College, Shengjing Hospital Affiliated to \\ China Medical University, Shenyang \\ I I0004, Liaoning, People's Republic of \\ China
}

Correspondence: Chunlin Ge Email gechunlin 126@126.com
Objective: To comprehensively analyze the global scientific outputs of nanoparticles in pancreatic cancer research.

Methods: Publications regarding the nanoparticles in pancreatic cancer research published from 1986 to 2019 were retrieved from the Web of Science Core Collection (WoSCC). Highly frequent keywords, publication years, journals, cited papers, cited journals and cited authors were identified using BICOMB software, and then a binary matrix and a co-word matrix were constructed. gCLUTO was used for double clustering of highly frequent journals. Co-citation analysis was performed using CiteSpace V software, including keywords, references, journals author or institution cooperation network.

Results: A total of 1171 publications were included in this study. Publications mainly came from 10 countries, led by the US $(n=470)$ and China $(n=349)$. Among the top 20 journals ranked by the number of citations, nanoscience nanotechnology was the leader with 300 . Cluster analysis of citation network identified 12 co-citation clusters, headed by "stromal barrier" and "emerging inorganic nanomaterial".

Conclusion: Our findings reveal the research performance and intellectual structure of the nanoparticles in pancreatic cancer research, which may help researchers understand the research trends and hotspots in this field.

Keywords: Web of Science, CiteSpace, pancreatic cancer, nanoparticles, co-citation analysis, co-word analysis

\section{Introduction}

Pancreatic cancer is a highly lethal disease with extremely poor prognosis. ${ }^{1,2}$ Patients with pancreatic cancer usually have a 5-year survival rate of less than $5 \%{ }^{3}$ Most patients with pancreatic cancer remain asymptomatic until the disease reaches an advanced stage. ${ }^{1,2}$ Surgical resection is the main treatment. ${ }^{4}$ Unfortunately, at the time of diagnosis, most patients are not suitable for surgical resection due to local advanced or distant metastases. Chemotherapy is the only approved non-surgical option for most patients with advanced pancreatic cancer, but it is rarely cured. Recently, it has been reported that energy-based methods such as high-intensity-focused ultrasound (HIFU) could reduce cancer pain and prolong the survival time of advanced pancreatic cancer patients. ${ }^{5}$ Pancreatic cancer has a high complexity in the tumor microenvironment, which leads to poor angiogenesis and severely limits the perfusion of the drug to the tumor after systemic administration. For example, epidermal growth factor receptor could a potential 
therapeutic target of monoclonal antibodies for pancreatic cancer therapy. ${ }^{6}$ Therefore, there is an urgent need for more effective treatments that focus on targeted delivery to overcome the enormous challenges posed by pancreatic cancer. Emerging research has been conducted on the application of nanotechnology principles for therapeutic drug delivery. Nano-systems can "escort" drug molecules to planned destinations, avoiding the body's natural barriers, preventing early degradation or metabolism of the drug. ${ }^{7-9}$ Nanocarriers are easy to synthesize and costeffective, which can be easily customized for the application. A number of studies have been conducted using a wide range of nanotechnology for the treatment of various cancers, including pancreatic cancer. ${ }^{10-12}$ So far, a few target nanomedicines have been approved and marketed such as DaunoXomeVR and Onco-TCSVR. ${ }^{13,14}$

However, there are few studies on the application of nanoparticles in pancreatic cancer using systematic bibliometric methods. Bibliometric analysis concerning the application of nanoparticles in pancreatic cancer has not been conducted, which is possible to provide more insight into how nanoparticles can play a role in the treatment of pancreatic cancer. Several bibliometric methods and tools have been developed to help researchers in different fields construct knowledge maps, assess collective thought states on topics and identify hot spots in the research field. The two most common methods include co-word analysis and co-citation analysis. Co-word analysis is an important bibliometric analysis method that helps researchers identify hot topics and trends in the discipline. ${ }^{15}$ In an article, if two words are often present at the same time, they may have a closer relationship than other pairs of words. ${ }^{16}$ References in an article can provide important insights into currently known information about a certain topic. The most common way to obtain this information is through co-citation analysis: the two papers cited in the third article have a co-citation relationship. Statistical analysis of the relationship strength between different articles can assist researchers determine the knowledge base of the discipline, research frontiers, key authors, and other bibliometric information. ${ }^{17,18}$ Because of the abstract nature of the information in the text, visual analysis is often utilized in bibliometric research via visualization tools such as CiteSpace and BICOMB. ${ }^{19,20}$ These tools can be used to perform bibliometric analysis of topics from a variety of perspectives, such as co-word analysis, cocitation analysis, and author and institutional collaboration analysis. For example, as shown in our previous study,
BICOMB was used to identify the hotspot distribution of subjects in the application of stents in pancreatic cancer by co-word analysis. ${ }^{21}$ Xiao et al analyzed the knowledge domain and emerging trends in organic photovoltaic technology using CiteSpace software. ${ }^{22}$ This study aimed to comprehensively analyze the current state of the application of nanoparticles in pancreatic cancer research from various angles using different bibliometric tools. Our findings clarified the intellectual structure and research performance in the field.

\section{Materials and Methods Data Source}

In September 2019, we accessed the WoSCC online database to identify publications with the following search strategies: topic = "nanoparticles" and "pancreatic cancer". To go into depth into the application of nanoparticles in the treatment of pancreatic cancer, we did not impose other restrictions on the search strategies, including publication years, publication types and publication languages.

\section{Data Extraction}

The searched publications were evaluated as the following perspectives: (1) publication years; (2) author institutions; (3) countries/regions; (4) journals; (5) WoS categories; (6) publication types; (7) cited numbers; (9) keywords.

\section{Co-Word Analysis}

TXT file was imported into co-occurrence matrix generation software (BICOMB) for data extraction and matrix construction. ${ }^{21}$ The frequency rankings of keywords, publication years, journals, cited papers, cited journals and authors were determined, respectively. According to $\mathrm{H}$ index, keywords, publication years, journals, cited papers, cited journals and authors were sorted in descending order of the frequency. Highly frequent keywords, publication years, journals, cited papers, cited journals and authors were identified. Furthermore, relationships between the highly frequent journals and the source literature were determined using biclustering analysis. A binary matrix was constructed based on the source literature set generated by BICOMB and the highly frequent journals as columns and rows. The results were visualized using "gCLUTO" software (version 1.0), a graphical cluster toolkit and graphical front-end of the "CLUTO" data clustering library. ${ }^{23}$ The cluster analysis was used to evaluate the highly frequent journals, including the bisection repeat, 
cosine as the similarity function, and $\mathrm{I} 2$ as the clustering criterion function. Through different numbers of clusters, two clusters were performed to differentiate the first-rank number of clusters.

\section{Co-Citation Analysis}

CiteSpace software (version 5.5.2) was used for cocitation analysis. CiteSpace is a bibliometric analysis visualization software developed by Chen. ${ }^{24}$ We imported the complete dataset of 1171 articles related to nanoparticles in pancreatic cancer into CiteSpace. The timespan was set as "2003-2019" and slice length was set as " 1 ". The 50 most cited papers for each time slice were selected, which were used to construct a co-citation network where each node represented a cited paper. After that, citation burst detection and timeline view were performed. Furthermore, dual-map overlay was performed to reveal overall scientific contributions. Finally, cited author collaboration and cited institution networks were constructed, respectively.

Full records of these publications and cited references were imported into VOSviewer (version 1.6.13) for highfrequent term and time fusion analyses. ${ }^{25}$ VOSviewer was used to extract and analyze terms in titles and abstracts of publications. After that, the association between extracted terms and citation counts was analyzed. Finally, the results were visualized into a bubble chart. Each bubble represented a term. The size of the bubble expressed the frequency of occurrence of the term (multiple occurrences in a publication count as one). If two words co-occurred more frequently, the two bubbles were closer. CitNetExplorer (version 1.0.0) was used to conduct citation networks, which could sort citation relationships of publications in a field in chronological order. ${ }^{26}$

\section{A}

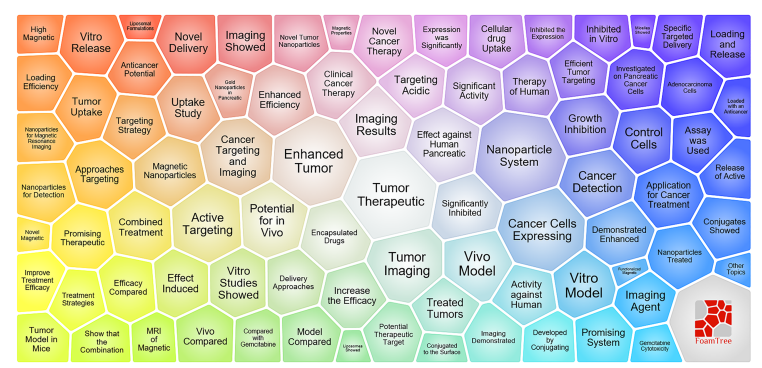

C

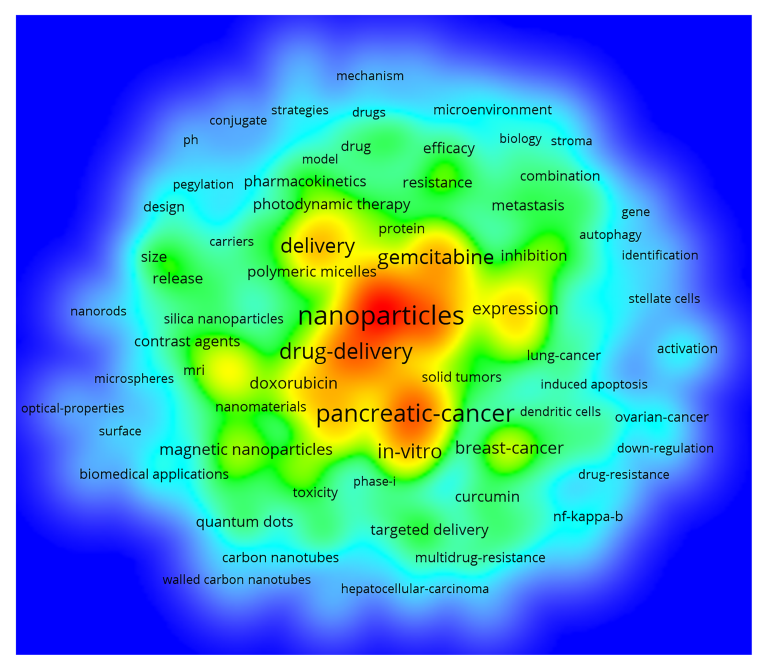

B
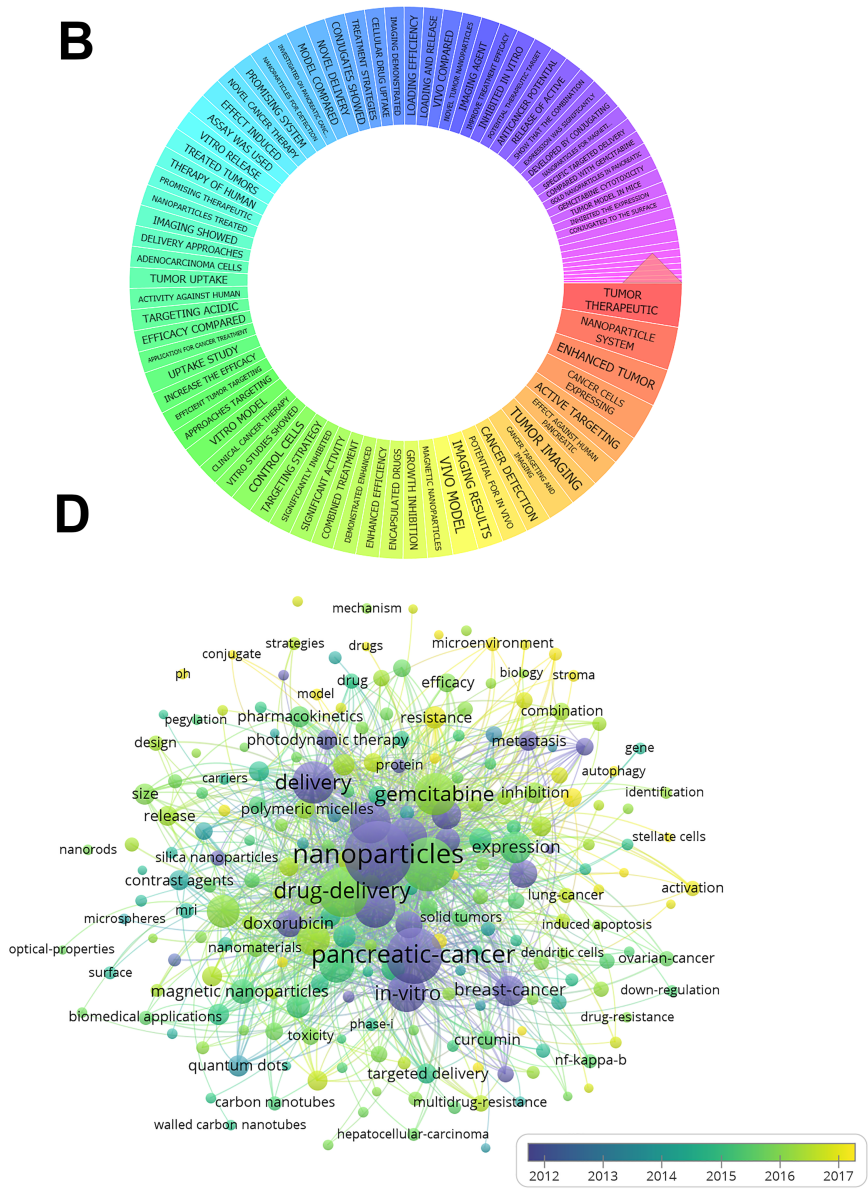

Figure I Major topics for the nanoparticles in pancreatic cancer. (A and B) Visualization of keyword ranking based on the carrot system. The larger the weight of the topic, the larger the area, and the more central the module. (C) Density visualization. (D) Keyword co-occurrence map visualization. Bubble chart visualized 219 keywords. The minimum number of occurrences of a keyword was set as 10 . The bigger the bubble, the higher the frequency. Furthermore, the closer the proximity, the higher the frequency. 
A

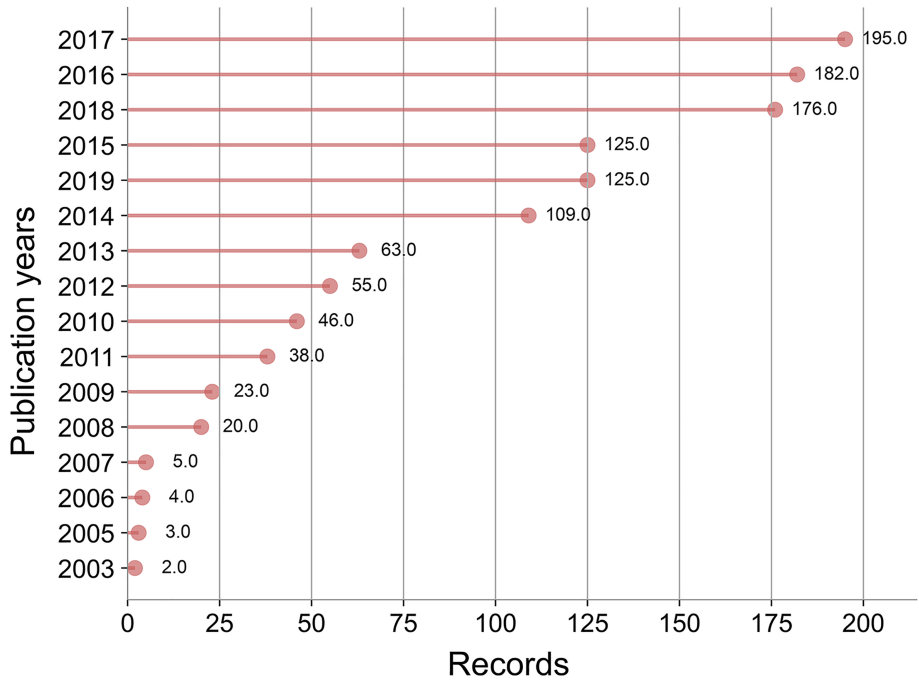

B

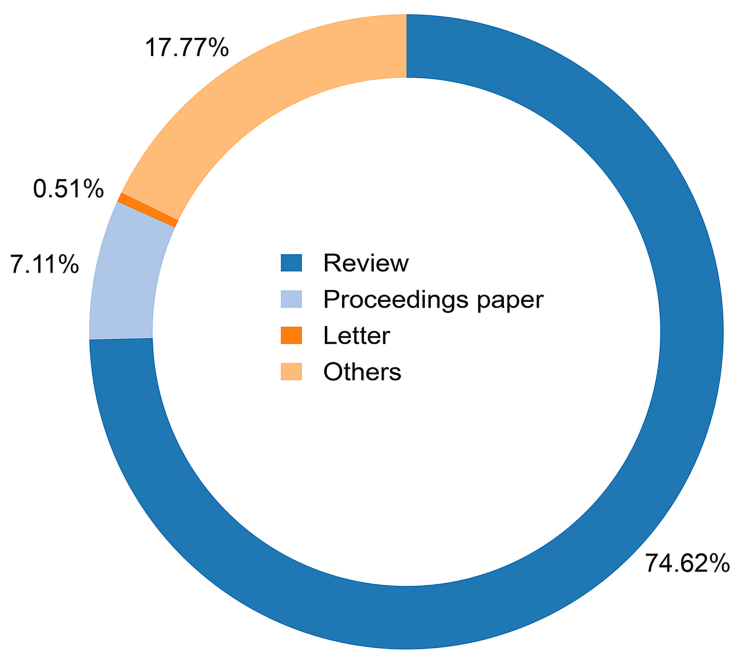

Figure 2 Publication years (A) and publication types (B).

\section{Results}

\section{Major Topic Surveys}

Major topics in the field of the nanoparticles in pancreatic cancer were analyzed using the carrot system and VOSviewer. For carrot2 results, we found that "tumor therapeutic", "nanoparticle system" and "enhanced tumor" were the top three topics (Figure 1A and B). As shown in Figure 1C, the main terms were as follows: "nanoparticles", "pancreatic cancer", "drug delivery" and "gemcitabine" according to VOSviewer. All keywords were mainly clustered into five groups. "Drug delivery" and "gemcitabine" occurred after "nanoparticles" and "pancreatic cancer" (Figure 1D).

\section{Distribution of Articles by Publication Years}

A total of 1171 relevant publications from 2003 to 2017 were collected for this study. As depicted in Figure 2A, the

Table I Highly Frequent Terms in the Nanoparticles in Pancreatic Cancer

\begin{tabular}{|l|l|l|l|l|}
\hline No. & Keywords & Frequency, $\mathbf{n}$ & Percentage, \% & Cumulative Percentage, \% \\
\hline 1 & Pancreatic cancer & 148 & 3.2132 & 3.2132 \\
2 & Pancreatic cancer & 113 & 2.4533 & 5.6665 \\
3 & Nanoparticles & 57 & 1.2375 & 6.9040 \\
4 & Nanoparticles & 56 & 1.2158 & 8.1198 \\
5 & Drug delivery & 56 & 1.2158 & 9.3356 \\
6 & Gemcitabine & 49 & 1.0638 & 10.3995 \\
7 & Drug delivery & 37 & 0.8033 & 11.2028 \\
8 & Gemcitabine & 35 & 11.9627 \\
9 & Cancer & 31 & 0.7599 & 12.6357 \\
10 & Nanomedicine & 25 & 0.6730 & 13.1785 \\
11 & Cancer & 25 & 0.5428 & 13.7212 \\
12 & Nanoparticle & 23 & 0.5428 & 14.2206 \\
13 & Nanoparticle & 22 & 0.4993 & 14.6982 \\
14 & Nanomedicine & 21 & 0.4776 & 15.1541 \\
15 & Magnetic resonance imaging & 20 & 0.4559 & 15.5884 \\
16 & Gold nanoparticles & 19 & 0.4342 & 16.0009 \\
17 & Apoptosis & 18 & 0.4125 & 16.3917 \\
18 & Nanotechnology & 17 & 0.3908 & 16.7607 \\
19 & Curcumin & 15 & 0.3691 & 17.0864 \\
20 & Gold nanoparticles & 15 & 0.3257 & 17.4121 \\
\hline
\end{tabular}


number of publications has been rising over time. In 2017 , 195 publications were addressed, suggesting that the field in 2017 was in a period of rapid growth. Among all publications, $83.177 \%$ were articles (Figure 2B).

\section{Highly Frequent Terms}

Keywords, publication year, journals, cited papers, cited journals and cited authors were extracted using BICOMB software. Highly frequent terms were then identified. The top 20 most frequent keywords are listed in Table 1, such as pancreatic cancer, nanoparticles, drug delivery and gemcitabine.

\section{Global Landscape}

The country distribution of the number of cited papers related to the nanoparticles in pancreatic cancer is depicted as Figure 3A. The results showed that US and China published the most papers, indicating that these two countries could be at the forefront of the world in this field.

\section{Highly Frequent Journals and Cited Journals}

We identified 20 highly frequent journals (Table 2) and cited journals (Table 3), therefore, focusing on articles published in these journals may help to understand the latest developments in the field. Highly frequent-cited papers in the nanoparticles in pancreatic cancer are listed in Table 4. "Burris HA, 1997, J CLIN ONCOL, V15, P2403", "Von Hoff DD, 2013, NEW ENGL J MED, V369, P1691”, “MATSUMURA Y, 1986, CANCER RES, V46, P6387" were the top three highly
A

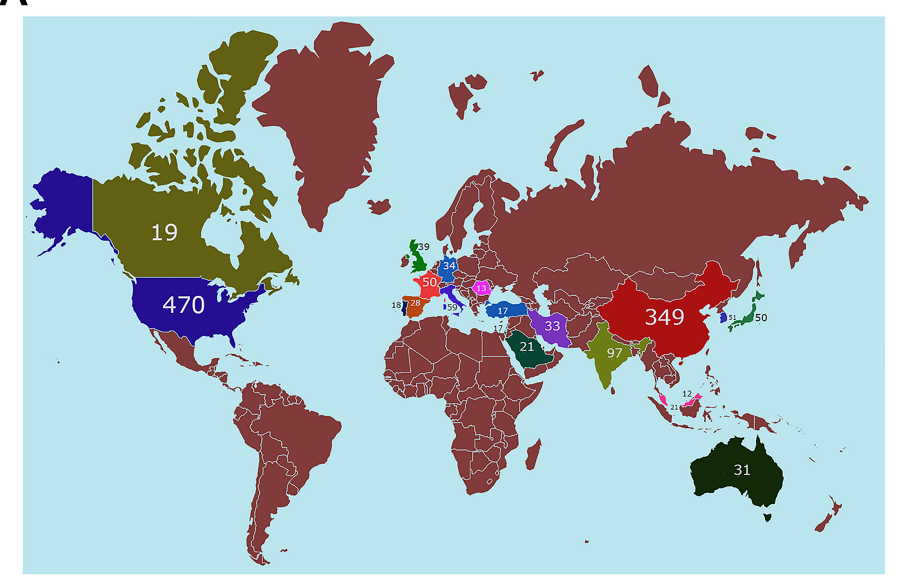

B

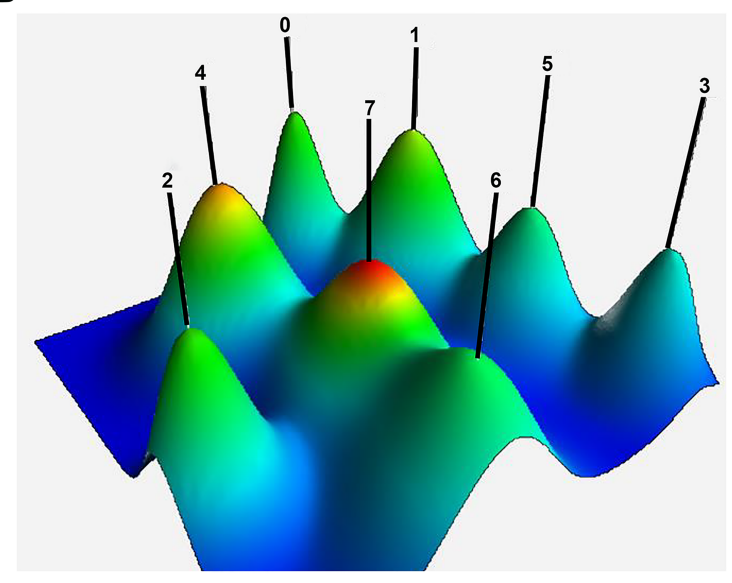

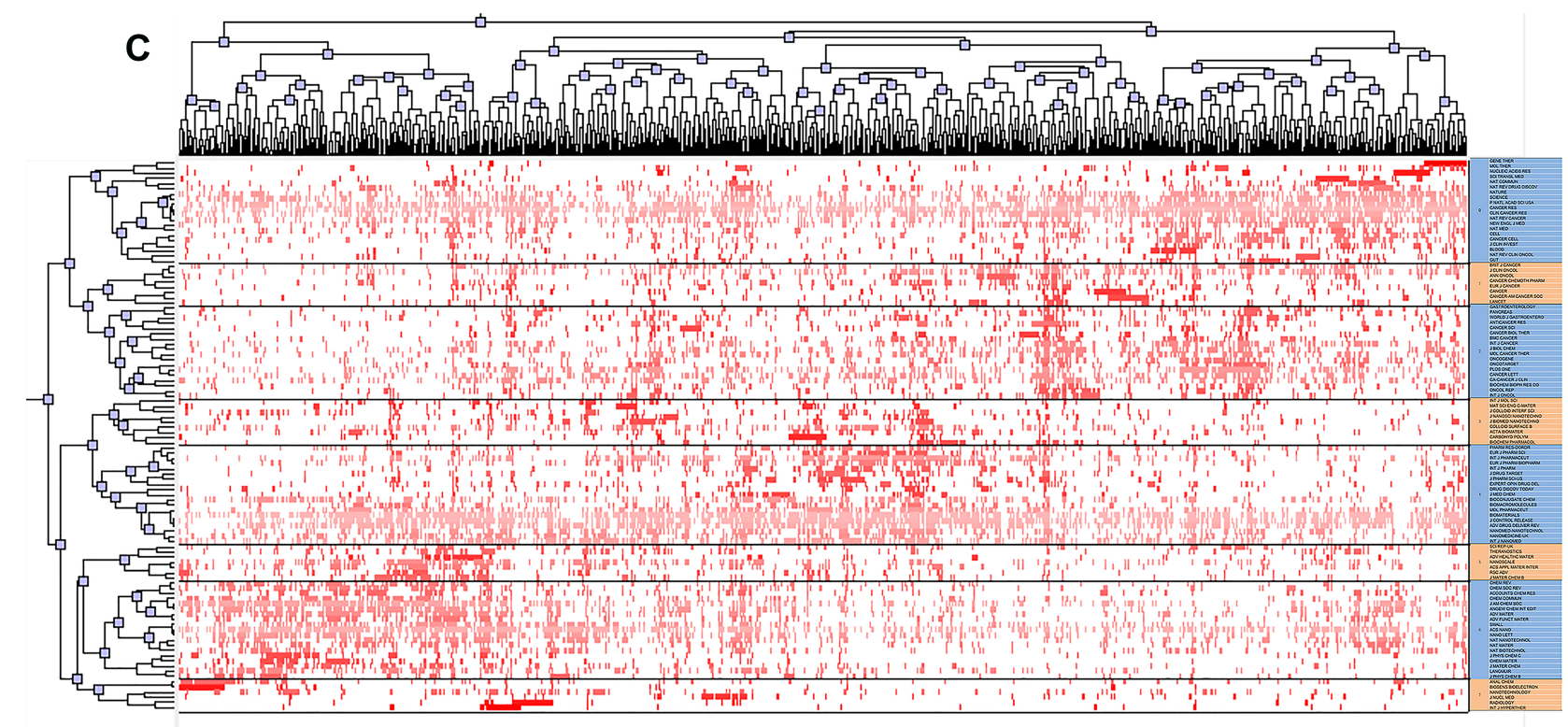

Figure 3 Clustering analysis of high frequently journals in the nanoparticles in pancreatic cancer. (A) The country distribution of cited papers related with the nanoparticles in pancreatic cancer. (B) A mountain visualization biclustering of highly frequent journals and articles on the nanoparticles in pancreatic cancer. (C) A visualized matrix biclustering of highly frequent journals and PubMed Unique Identifiers of articles related with the nanoparticles in pancreatic cancer. 
Table 2 Highly Frequent Journals in the Nanoparticles in Pancreatic Cancer

\begin{tabular}{|l|l|l|l|l|}
\hline No. & Journals & Frequency, $\mathbf{n}$ & Percentage, \% & Cumulative Percentage, \% \\
\hline I & Journal of Controlled Release & 45 & 3.8429 & 3.8429 \\
2 & International Journal of Nanomedicine & 45 & 3.8429 & 7.6857 \\
3 & ACS nano & 44 & 3.7575 & 11.4432 \\
4 & Biomaterials & 39 & 3.3305 & 14.7737 \\
5 & RSC Advances & 27 & 2.3057 & 17.0794 \\
6 & International Journal of Pharmaceutics & 24 & 2.0495 & 19.1289 \\
7 & Journal of Materials Chemistry B & 23 & 1.9641 & 21.0931 \\
8 & ACS Applied Materials \& Interfaces & 23 & 1.9641 & 23.0572 \\
9 & Nanomedicine-Nanotechnology Biology and Medicine & 23 & 1.9641 & 25.0213 \\
10 & Cancer Research & 23 & 1.9641 & 26.9855 \\
11 & Theranostics & 19 & 1.6225 & 28.6080 \\
12 & Molecular Pharmaceutics & 18 & 1.5371 & 30.1452 \\
13 & Nanoscale & 16 & 1.3664 & 31.5115 \\
14 & Scientific Reports & 16 & 1.3664 & 32.8779 \\
15 & Journal of Biomedical Nanotechnology & 15 & 1.2810 & 34.1588 \\
16 & Colloids and Surfaces B-Biointerfaces & 14 & 1.1956 & 35.3544 \\
17 & Small & 14 & 1.1956 & 36.5500 \\
18 & PLoS One & 14 & 1.1956 & 37.7455 \\
19 & Nanomedicine & 14 & 1.1956 & 38.9411 \\
20 & Bioconjugate Chemistry & 13 & 40.0512 \\
\hline
\end{tabular}

Table 3 Highly Frequent Cited Journals in the Nanoparticles in Pancreatic Cancer

\begin{tabular}{|l|l|l|l|l|}
\hline No. & Journals & Frequency, $\mathbf{n}$ & Percentage, \% & Cumulative Percentage, \% \\
\hline 1 & Biomaterials & 1918 & 2.9466 & 2.9466 \\
2 & J Control Release & 1899 & 2.9175 & 5.8641 \\
3 & Cancer Res & 1755 & 2.6962 & 8.5603 \\
4 & Acs nano & 1393 & 2.1401 & 10.7004 \\
5 & P Natl Acad Sci USA & 1083 & 1.6638 & 12.3642 \\
6 & Adv Drug Deliver Rev & 1048 & 1.6101 & 13.9743 \\
7 & J Am Chem Soc & 1038 & 1.5947 & 15.5690 \\
8 & Clin Cancer Res & 915 & 1.4057 & 16.9747 \\
9 & Int J Nanomed & 753 & 1.1568 & 18.1315 \\
10 & INT J PHARMACEUT & 703 & 1.0800 & 19.2116 \\
11 & Nano Lett & 653 & 1.0032 & 20.2148 \\
12 & J Clin Oncol & 608 & 0.9341 & 21.1489 \\
13 & Science & 601 & 0.9233 & 22.0722 \\
14 & Nature & 600 & 0.9218 & 22.9940 \\
15 & Mol Pharmaceut & 571 & 0.8772 & 23.8712 \\
16 & PLoS One & 556 & 0.8542 & 24.7254 \\
17 & Nat Rev Cancer & 529 & 0.8127 & 25.5381 \\
18 & Bioconjugate Chem & 522 & 0.8020 & 26.3400 \\
19 & Angew Chem Int Edit & 489 & 0.7513 & 27.0913 \\
20 & Small & 480 & 0.7374 & 27.8287 \\
\hline
\end{tabular}

cited papers. These highly cited articles may be the cornerstone of the field.

Highly frequent journals were categorized into eight clusters, reflecting the general outline of the research development of the discipline (Figure 3B). Descriptive and discriminating features in the eight clusters are listed in Table 5. By analyzing the cited journals within each cluster, the main knowledge structure and research status in the nanoparticles in pancreatic cancer field could be determined. Highly frequent journals were distinguished into eight 
Table 4 Highly Frequent Cited Papers in the Nanoparticles in Pancreatic Cancer

\begin{tabular}{|c|c|c|c|c|}
\hline No. & Articles & Frequency, n & Percentage, \% & Cumulative Percentage, $\%$ \\
\hline I & Burris HA, 1997, J Clin Oncol, VI5, P2403 & 66 & 0.1014 & 0.1014 \\
\hline 2 & Von Hoff DD, 2013, New Engl J Med, V369, PI69I & 62 & 0.0953 & 0.1966 \\
\hline 3 & Matsumura Y, 1986, Cancer Res, V46, P6387 & 61 & 0.0937 & 0.2904 \\
\hline 4 & Peer D, 2007, Nat Nanotechnol, V2, P75I & 56 & 0.0860 & 0.3764 \\
\hline 5 & Cabral H, 20II, Nat Nanotechnol, V6, P8I5 & 55 & 0.0845 & 0.4609 \\
\hline 6 & Olive KP, 2009, Science, V324, PI457 & 49 & 0.0753 & 0.5362 \\
\hline 7 & Jain RK, 2010, Nat Rev Clin Oncol, V7, P653 & 47 & 0.0722 & 0.6084 \\
\hline 8 & Lee GY, 20I3, ACS nano, V7, P2078 & 44 & 0.0676 & 0.6760 \\
\hline 9 & Patra CR, 2008, Cancer Res, V68, PI970 & 44 & 0.0676 & 0.7436 \\
\hline 10 & Maeda H, 2000, J Control Release, V65, P27I & 42 & 0.0645 & 0.8081 \\
\hline II & Provenzano PP, 2012, Cancer Cell, V2I, P4I8 & 42 & 0.0645 & 0.8726 \\
\hline 12 & Conroy T, 20II, New Engl J Med, V364, PI8I7 & 41 & 0.0630 & 0.9356 \\
\hline 13 & Meng H, 20I5, ACS nano, V9, P3540 & 41 & 0.0630 & 0.9986 \\
\hline 14 & Hidalgo M, 2010, New Engl J Med, V362, PI605 & 40 & 0.0615 & 1.0601 \\
\hline 15 & Davis ME, 2008, Nat Rev Drug Discov, V7, P77I & 39 & 0.0599 & 1.1200 \\
\hline 16 & Ferrari M, 2005, Nat Rev Cancer, V5, PI6I & 36 & 0.0553 & 1.1753 \\
\hline 17 & Fang J, 20II, Adv Drug Deliver Rev, V63, PI36 & 35 & 0.0538 & 1.2290 \\
\hline 18 & Erkan M, 20I2, Nat Rev Gastro Hepat, V9, P454 & 32 & 0.0492 & 1.2782 \\
\hline 19 & Li DH, 2004, Lancet, V363, PI049 & 31 & 0.0476 & 1.3258 \\
\hline 20 & Siegel R, 20I4, CA-Cancer J Clin, V64, P9 & 31 & 0.0476 & 1.3735 \\
\hline
\end{tabular}

Table 5 Descriptive and Discriminating Features in the Eight Clusters

\begin{tabular}{|l|l|l|l|l|l|}
\hline Cluster & Size & Internal Similarity & Internal Standard Deviation & External Similarity & External Standard Deviation \\
\hline 0 & 7 & 0.386 & 0.038 & 0.180 & 0.030 \\
1 & 19 & 0.380 & 0.046 & 0.181 & 0.049 \\
2 & 8 & 0.350 & 0.039 & 0.176 & 0.041 \\
3 & 6 & 0.290 & 0.020 & 0.129 & 0.034 \\
4 & 19 & 0.372 & 0.060 & 0.211 & 0.063 \\
5 & 9 & 0.293 & 0.026 & 0.157 & 0.025 \\
6 & 18 & 0.304 & 0.029 & 0.180 & 0.034 \\
7 & 20 & 0.315 & 0.069 & 0.191 & 0.066 \\
\hline
\end{tabular}

clusters in matrix visualization (Figure 3C). The top and left of hierarchical tree, respectively, showed the associations between highly frequent journals and relevant papers. Furthermore, the corresponding paper was obviously shown for each highly frequent journal in each cluster.

\section{Highly Frequent Cited Author}

We also focused on highly frequent-cited authors, such as "Yong, KT", "Wang, J" and "Zhang, Y" (Table 6). Highly cited authors were pioneers in the field and could significantly contribute to the development of the field.

\section{Citation Bursts}

The literature represented in the co-citation network was clustered into 12 categories (Figure 4A and B). The modularity was 0.688 , indicating that the specialties were clearly defined in terms of co-citation clusters. However, the mean silhouette score was 0.3201 due to the numerous small clusters. The categories were tagged by keywords of the cited papers. Through comprehensively analyzing the tags and the papers corresponding to each category of nodes, the main intellectual structure and the current state of research in the application of nanoparticles in pancreatic cancer can be determined.

Major milestones in the development of the nanoparticles in pancreatic cancer can be identified from the list of references that had strong citation bursts from 2003 to 2019 (Figure 4C). CiteSpace software was used to analyze the co-citation network. The top 20 citation bursts were identified. The increasing number of citations of these 
Table 6 Highly Frequent Cited Authors in the Nanoparticles in Pancreatic Cancer

\begin{tabular}{|l|l|l|l|l|}
\hline No. & Authors & Frequency, $\mathbf{n}$ & $\begin{array}{l}\text { Percentage, } \\
\%\end{array}$ & $\begin{array}{l}\text { Cumulative } \\
\text { Percentage, \% }\end{array}$ \\
\hline 1 & Yong, KT & 29 & 0.3458 & 0.3458 \\
2 & Wang, J & 23 & 0.2742 & 0.6200 \\
3 & Zhang, Y & 17 & 0.2027 & 0.8227 \\
4 & Roy, I & 17 & 0.2027 & 1.0254 \\
5 & Couvreur, P & 16 & 0.1908 & 1.2162 \\
6 & Prasad, PN & 16 & 0.1908 & 1.4069 \\
7 & Wang, Y & 14 & 0.1669 & 1.5739 \\
8 & Yallapu, MM & 14 & 0.1669 & 1.7408 \\
9 & Jaggi, M & 14 & 0.1669 & 1.9077 \\
10 & Kataoka, K & 14 & 0.1669 & 2.0746 \\
11 & Chauhan, SC & 14 & 0.1669 & 2.2416 \\
12 & Huang, L & 13 & 0.1550 & 2.3966 \\
13 & Yang, L & 13 & 0.1550 & 2.5516 \\
14 & Fu, DL & 13 & 0.1550 & 2.7066 \\
15 & Li, J & 13 & 0.1550 & 2.8616 \\
16 & Khan, S & 13 & 0.1550 & 3.0166 \\
17 & Hu, R & 12 & 0.1431 & 3.1597 \\
18 & Mao, H & 12 & 0.1431 & 3.3027 \\
19 & Wang, LY & 12 & 0.1431 & 3.4458 \\
20 & Jin, C & 12 & 0.1431 & 3.5889 \\
\hline
\end{tabular}

papers in a certain period indicated the rapid acceptance and dissemination in the application of nanoparticles in pancreatic cancer. These papers could contribute to the knowledge structure in the field.

\section{Timeline View from 2000 to 2018}

A timeline view can depict changes in the trends of a field with time. In this study, the timeline was visualized using CiteSpace. In Figure 5, nodes of a cluster shared a horizontal line. The publication dates of papers were at the top of the view, while the latest articles were on the right. The timeline view clearly described the number of nodes in each cluster. The number of nodes in the cluster reflected the importance of this topic in the field. The greater the number of nodes, the more important it was. This timeline view visualized the most important articles in a particular topic and showed the emergence, popularity and decline of research topics. Furthermore, the view also showed the temporal characteristics of the study area reflected by the cluster. As depicted in Figure 5, \#cluster 0 "stromal barrier" and \#cluster 1 "emerging inorganic nanomaterial" occurred first. \#cluster 9 "targeted genetic medicine" and \#cluster 11 "nano-quantitative structure-activity" developed late.

\section{Discipline and Journal Analysis}

A dual-map overlay of the nanoparticles in pancreatic cancer research literature stood for the entire dataset in the nanoparticles in pancreatic cancer generated from journals indexed on the Web of Science. The dual-map overlay visualized the citation state of the nanoparticles in pancreatic cancer research. As depicted in Figure 6A, the left half side suggested the citing map, and the right half side represented the cited map. Furthermore, the curve was the citation path connection line from the left side to the right side. This connection demonstrated the flow of knowledge and connections in different areas of research. The dual-map overlay showed that the nanoparticles in pancreatic cancer papers were published in various disciplines. Figure $6 \mathrm{~B}$ shows the top 20 journals according to cited records in the field.

\section{Citation Networks}

CiteNetExplorer was used to construct citation networks for the nanoparticles in pancreatic cancer. We analyzed the 132 most cited publications from 2003 to 2017 (Figure 7A). The citation network consisted of 330 citation links. Furthermore, we also analyzed the 10 most cited publications from 2008 to 2012. There were 20 citation links in Figure 7B.

\section{Co-Cited Publication Cluster Analysis}

Figure 8A depicts the density map of the co-citation publications, which could provide the intellectual structure for the nanoparticles in pancreatic cancer field. Moreover, a co-cited publication network was visualized (Figure 8B). As shown in Figure $8 \mathrm{~A}$ and $\mathrm{B}$, four co-cited publication clusters were conducted, which were expressed in different colors.

\section{Co-Citation Map of Authors and Institutions on Clustered Networks}

CiteSpace was used to analyze the cited author cooperation network regarding the nanoparticles in pancreatic cancer research. The results showed the top 20 cited authors. As shown in Figure 9A, the network had a modularity of 0.6793 , which was relatively high. The mean silhouette score was 0.4633 which was relatively low due to numerous small clusters. Among the top 20 most cited authors, HEINEMANN $\mathrm{V}$, ZHANG Y, REDDY LH, MAEDA $\mathrm{H}$ had the greatest citation numbers per publication. We next analyzed a total of 696 institutions participating in the nanoparticles in pancreatic cancer research using CiteSpace. The top 50 institutions were identified, as shown in Figure 9B. The network had a modularity of 0.688 , which was relatively high. Moreover, 

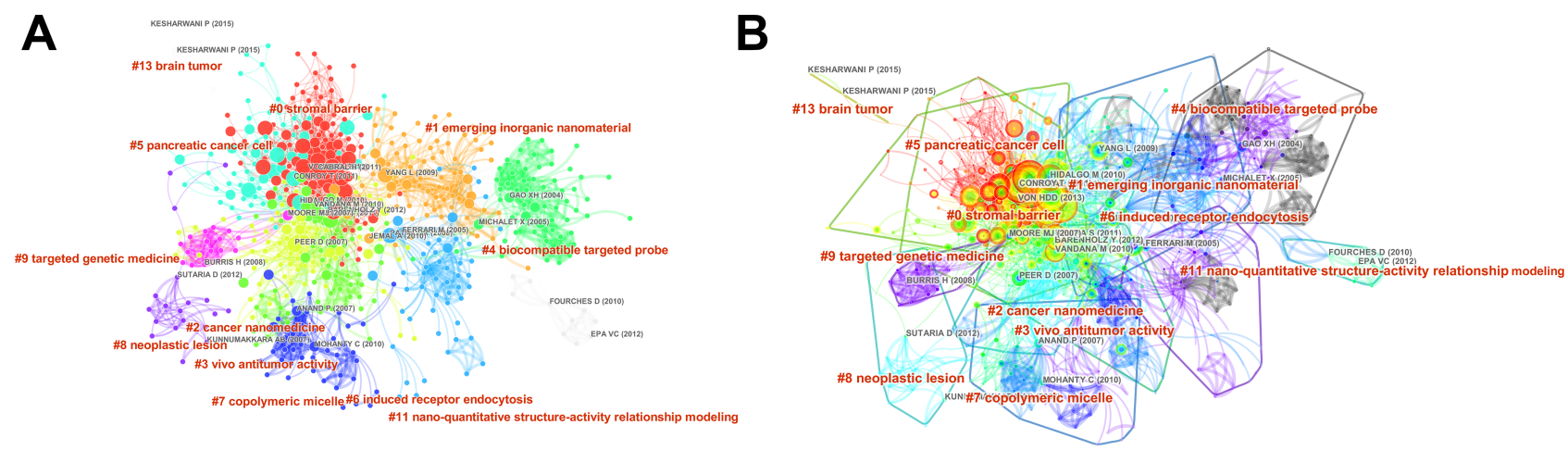

C

Top 20 References with the Strongest Citation Bursts



Figure 4 Citation burst. (A and B) Clustering analysis of the nanoparticles in pancreatic cancer co-citation network, generated by top 50 per slice between 1986 and 2019. $(\mathrm{LRF}=3.0, \mathrm{LBY}=8$, and $\mathrm{e}=2.0$ ). The size of the node represents the number of times of the cited literature, and the color of the line expresses the time when an article is first cited. Red text stands for the terms extracted from the title of the citing article in each cluster. (C) The top 20 references with the strongest citation bursts in the cocitation network.

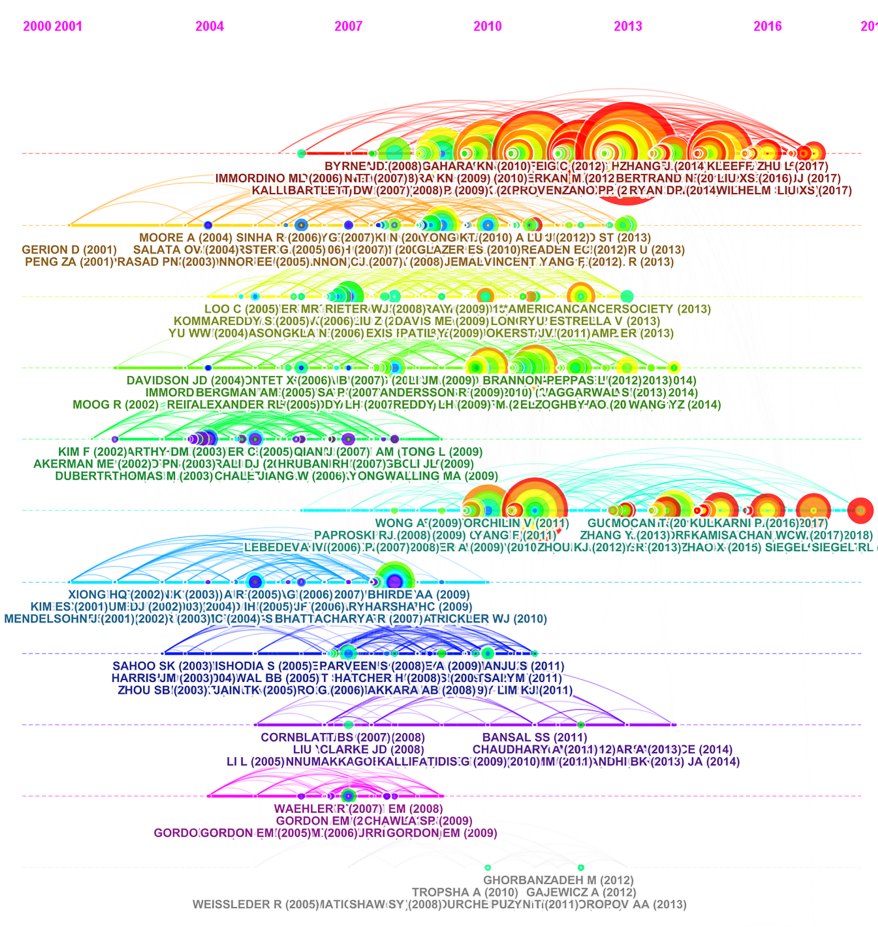

\section{\#0 stromal barrier}

\#1 emerging inorganic nanomaterial

\#2 cancer nanomedicine

\#3 vivo antitumor activity

\#4 biocompatible targeted probe

\#5 pancreatic cancer cell

\#6 induced receptor endocytosis

\#7 copolymeric micelle

\#8 neoplastic lesion

\#9 targeted genetic medicine

\#11 nano-quantitative structure-activity relationship \#13 brain tumor

Figure 5 A timeline visualization from 2000 to 2018. Nodes are labeled with corresponding topics. 


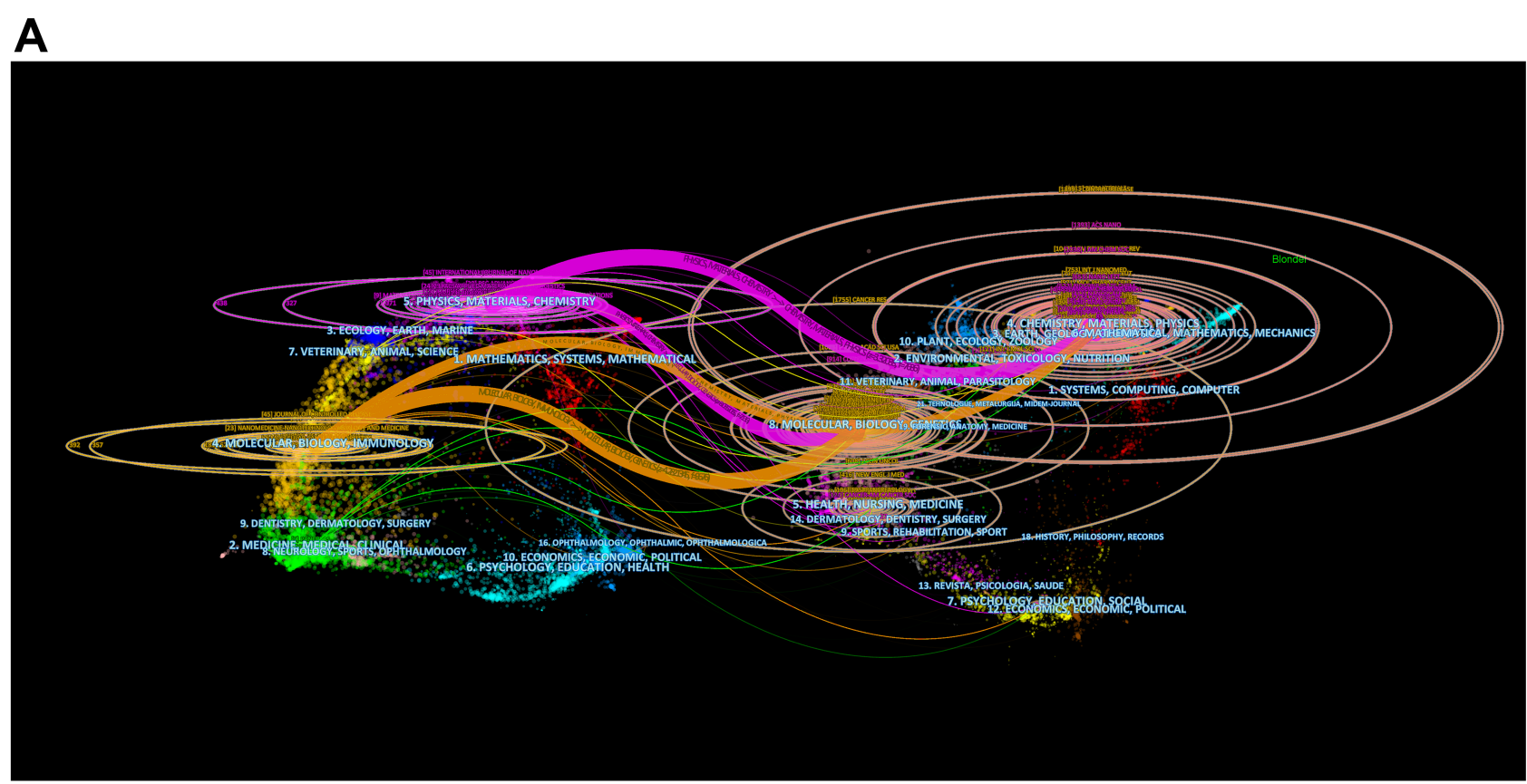

B

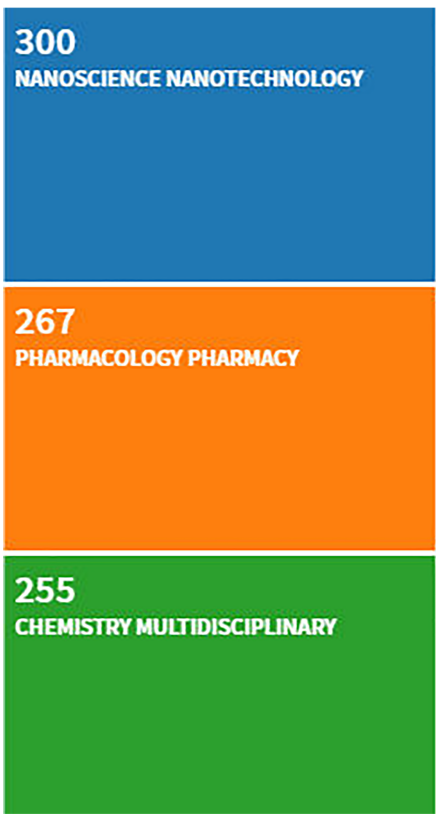

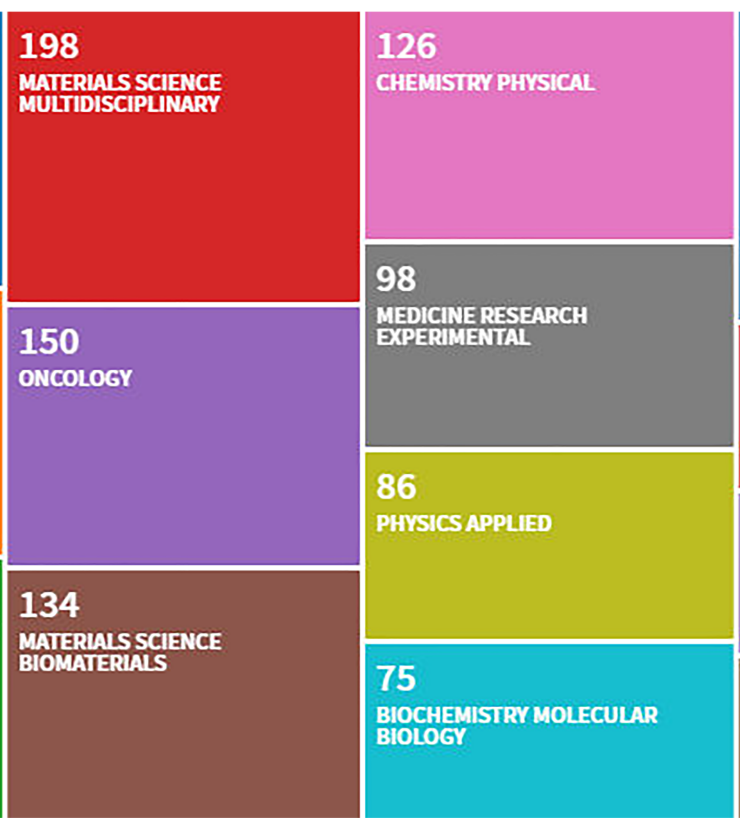

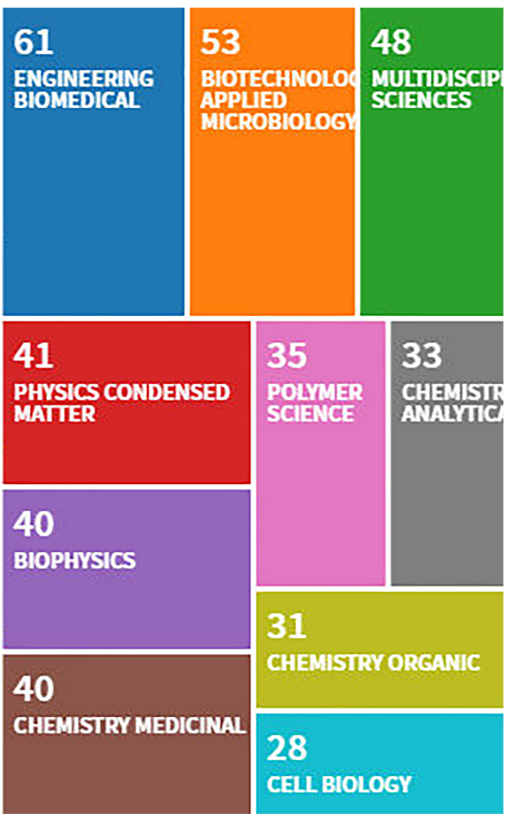

Figure 6 Discipline and journal analysis. (A) Dual-map overlap of the nanoparticles in pancreatic cancer literature. The colored curve suggests the reference path. The nature of each region is defined by the Journal of the corresponding region. (B) The top 20 journals according to cited records in the field of the nanoparticles in pancreatic cancer.

the mean silhouette score was 0.3201 which was relatively low due to numerous small clusters. These most cited institutions had the strong cooperation.

\section{Discussion}

This study explored the research performance of the nanoparticles in pancreatic cancer research by applying different bibliometric tools. For the first time, we analyzed the application of nanoparticles in pancreatic cancer from a bibliometric perspective and extended the application scope of bibliometric analysis. Through combining coword analysis and co-citation analysis, we identified the knowledge base and development status of the nanoparticles in pancreatic cancer research. We found that the most important intellectual basis of the nanoparticles in pancreatic cancer was composed of tumor therapeutic, 
A

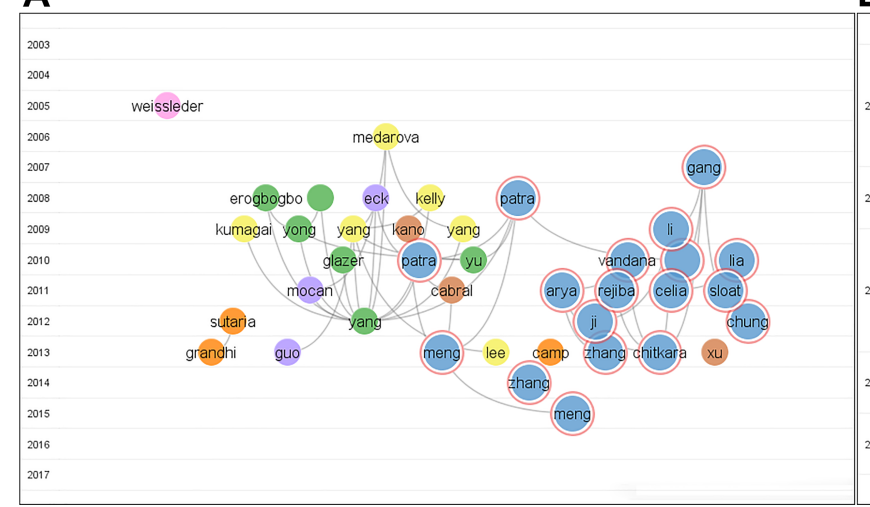

B

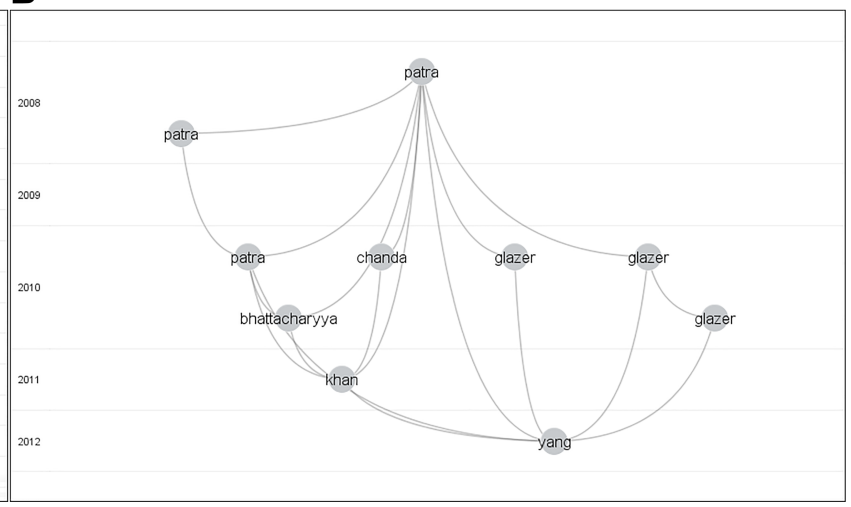

Figure 7 Citation networks. (A) Citation network of the 132 most cited publications from 2003 to 2017. (B) Citation network of the 10 most cited publications from 2008 to 2012. The curves between the nodes suggest the citation relationships.

\section{A}

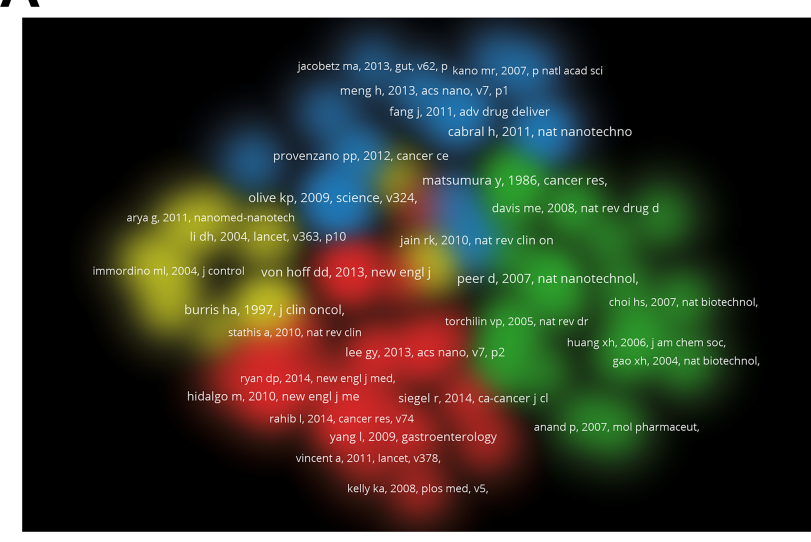

B

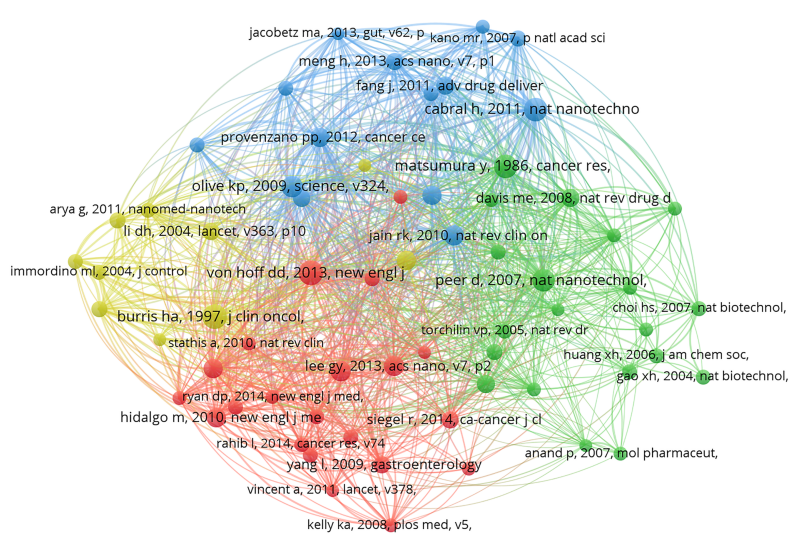

Figure 8 Co-cited publication cluster analysis. (A) Co-cited publication density map based on co-citation. (B) Co-cited publication network.

A

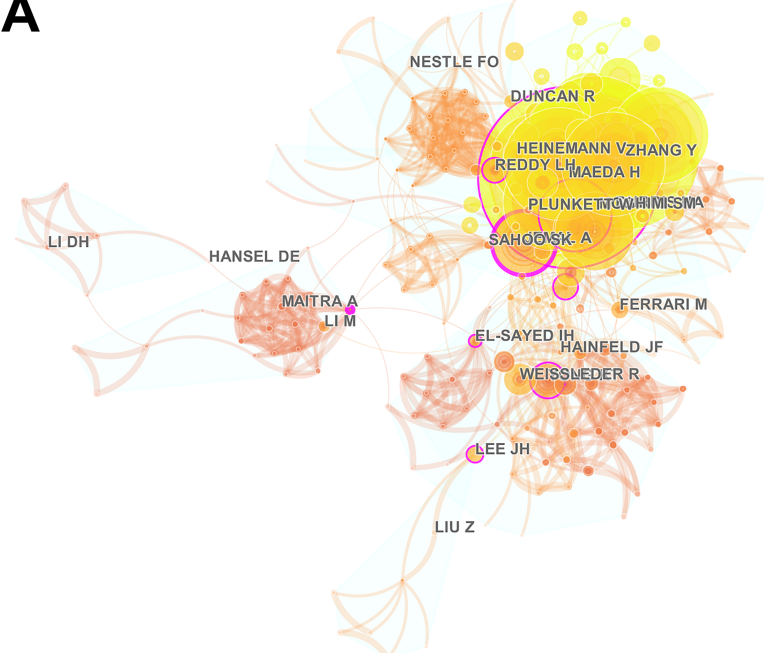

B

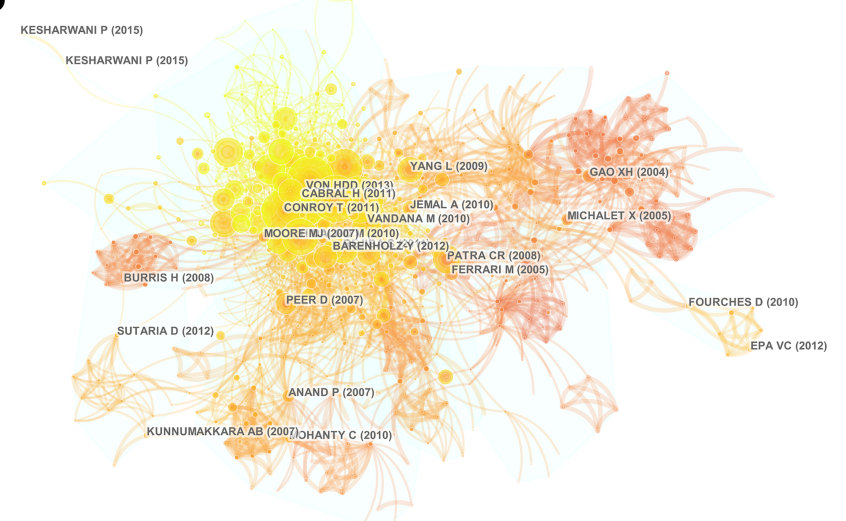

Figure 9 Co-citation map of authors and institutions on clustered networks. Co-cited author network (A) and co-cited institution network (B), generated by top 20 per slice between 1986 and $2019(\mathrm{LRF}=3.0, \mathrm{LBY}=8$, and $\mathrm{e}=2.0)$. The size of the nodes in the network indicates the amount of publications by authors or institutions. 
enhanced tumor and nanoparticle system over the past decade. ${ }^{27-29}$ Although most of the research on the nanoparticles in pancreatic cancer is carried out in isolation, there is little attempt to combine the comprehensive study of the nanoparticles in pancreatic cancer with other disciplines. Therefore, there is still an encouraging trend in the nanoparticles in pancreatic cancer.

We conducted co-word analysis to visually present the hotspots of the nanoparticles in the pancreatic cancer research. Co-word analysis is a micro-analysis method, which is used to determine the research hotspots of a certain topic and to visually reveal the findings. ${ }^{30-32}$ Furthermore, the citations of the article are also of reference value because they indirectly reflect important concepts of a particular topic. Thus, in this study, co-citation analysis was utilized to identify major knowledge bases for the nanoparticles in pancreatic cancer research.

We found that emerging inorganic nanomaterial is a research hotspot and an important knowledge base in this field. ${ }^{33}$ The combination of the time zone view and the reference burst detection indicated that the PRASAD PN 2004 had the greatest impact on the magnetic field, with the largest detonation burst strength being 4.9608. This article was also the earliest landmark node in the time zone view, followed by other important nodes. Therefore, the PRASAD PN 2004 study could be considered as a "trigger study," which could trigger other major breakthroughs or scientific activities. This type of work can facilitate further research and publications, making it a groundbreaking study in the field. The co-citation network showed the importance of article published by YANG L (2009) and had more co-citation links than other published articles in the nanoparticles in pancreatic cancer. ${ }^{34}$ Therefore, the article may play a pivotal role in the process of inheriting and spreading knowledge. In addition to the innovative academic significance, the novelty and diversity of research content can also explain the importance of these works. Furthermore, time zone view analysis showed that, in recent years, the research focus of the nanoparticles in pancreatic cancer has been "stromal barrier", "emerging inorganic nanomaterial" and "cancer nanomedicine". Among them, stromal barrier is the main feature of pancreatic cancer, including cellular components (such as fibroblasts, immune cells and endothelial cells), acellular components (such as collagen, fibronectin, growth factors and cytokines) and biophysical components (such as low $\mathrm{pH}$ and low oxygen). ${ }^{12}$ This may be due to the slow progress of the nanoparticles in pancreatic cancer. From the time zone view, we found that very few important nodes at the end of the time zone. However, this is to be expected because co-citation and citation analysis are not sensitive to the latest research, and no follow-up article can refer to the work. In the dual-map overlay analysis, we determined that the nanoparticles in pancreatic cancer could be limited to the fields of medicine and materials chemistry, but few studies are related to other disciplines. Therefore, the interdisciplinary study of the nanoparticles in pancreatic cancer may be a potential development opportunity.

As shown in our timeline view of the nanoparticles in pancreatic cancer co-citation network, "targeted genetic medicine" and "nano-quantitative structure-activity" developed late. $^{27,35}$ Targeted genetic medicine may be helpful to monitor, detect, and cure cancer effectively, which provides safe and effective personalized treatments for cancer patients by specifically targeting and preventing cancer cells in the body. Furthermore, targeted nano-medicine only needs a low dose of the drug, which reduces drug toxicity and side effects. Nanoparticles provide an ideal platform for integrating desired properties into a single gene delivery system and have a wide range of applications in controlling drug release and targeting cancer cells. Targeted genetic medicine encapsulated by nanoparticles can be a promising therapy for pancreatic cancer. ${ }^{36}$ Since experimental evaluation of the safety of nanoparticles is expensive and time consuming, it has been found that several computational methods, particularly nano-quantitative structure-activity relationships (it refers to the relationships between the measured cellular uptakes of nanoparticles in pancreatic cancer cells and their physical, chemical and structural properties.), are an effective alternative to predicting the potential toxicity of new nanoparticles prior to mass production. ${ }^{35}$

The vigorous development of nanotechnology has a huge impact on the medical field. The application of drug delivery with nanoparticles greatly helps to reduce the mortality of cancer. Compared to conventional treatments (such as chemotherapy and radiotherapy), drug delivery with nanoparticles may effectively avoid high doses of drug, systemic toxicity, damage of normal cells and lack of potential targeted output. ${ }^{37}$ Furthermore, there are other advantages of nanoparticles for cancer treatment. For example, nanoparticles can be used to enhance heating mechanism of energy-based method such as HIFU. ${ }^{38}$ Thus, drug delivery with nanoparticles has the potential for improving therapeutic efficacy for pancreatic cancer. In this study, we showed the research performance and intellectual structure of the nanoparticles in pancreatic cancer research, which may help researchers understand the research trends and hotspots in this field. 


\section{Conclusion}

Co-word and co-citation analyses were performed using various scientific metrology tools to reveal the bibliometric characteristics of the nanoparticles in the pancreatic cancer field. In this study, we identified the research development in the field and evaluated the publications of research institutions to guide research strategies and fund decision-making.

\section{Abbreviations}

WoSCC, Web of Science Core Collection; BICOMB, cooccurrence matrix generation software.

\section{Data Sharing Statement}

The datasets analyzed during the current study are available from the corresponding author on reasonable request.

\section{Author Contributions}

All authors made substantial contributions to conception and design, acquisition of data, or analysis and interpretation of data; took part in drafting the article or revising it critically for important intellectual content; gave final approval of the version to be published; and agree to be accountable for all aspects of the work.

\section{Funding}

This work was funded by Natural Science Foundation of Liaoning Province (2020-BS-283) and the project was funded by China Postdoctoral Science Foundation (2020M670818).

\section{Disclosure}

The authors declare no conflicts of interest.

\section{References}

1. Collisson EA, Bailey P, Chang DK, Biankin AV. Molecular subtypes of pancreatic cancer. Nat Rev Gastroenterol Hepatol. 2019;16 (4):207-220. doi:10.1038/s41575-019-0109-y

2. Wolfgang CL, Herman JM, Laheru DA, et al. Recent progress in pancreatic cancer. CA Cancer J Clin. 2013;63(5):318-348. doi:10. $3322 /$ caac. 21190

3. Singh A, Xu J, Mattheolabakis G, Amiji M. EGFR-targeted gelatin nanoparticles for systemic administration of gemcitabine in an orthotopic pancreatic cancer model. Nanomedicine: NBM. 2016;12 (3):589-600. doi:10.1016/j.nano.2015.11.010

4. Eberlin LS, Margulis K, Planell-Mendez I, et al. Pancreatic cancer surgical resection margins: molecular assessment by mass spectrometry imaging. PLoS Med. 2016;13(8):e1002108. doi:10.1371/journal. pmed.1002108

5. Zhu B, Li J, Diao L, Ma K, Fan Y, Yang W. High-intensity focused ultrasound ablation for advanced pancreatic cancer. $J$ Cancer Res Ther. 2019;15(4):831-835. doi:10.4103/jcrt.JCRT_408_18
6. Habban Akhter M, Sateesh Madhav N, Ahmad J. Epidermal growth factor receptor based active targeting: a paradigm shift towards advance tumor therapy. Artif Cells Nanomed Biotechnol. 2018;46 (sup2):1188-1198. doi:10.1080/21691401.2018.1481863

7. Grunwald B, Vandooren J, Locatelli E, et al. Matrix metalloproteinase-9 (MMP-9) as an activator of nanosystems for targeted drug delivery in pancreatic cancer. $J$ Control Release. 2016;239:39-48. doi:10.1016/j.jconrel.2016.08.016

8. Grunwald B, Vandooren J, Locatelli E, et al. Corrigendum to "Matrix metalloproteinase-9 (MMP-9) as an activator of nanosystems for targeted drug delivery in pancreatic cancer" [J. Control. Release 239 (2016) 39-48]. J Control Release. 2017;261:367. doi:10.1016/j. jconrel.2017.07.027

9. Rebelo A, Molpeceres J, Rijo P, Reis CP. Pancreatic cancer therapy review: from classic therapeutic agents to modern nanotechnologies. Curr Drug Metab. 2017;18(4):346-359. doi:10.2174/138920021 8666170201151135

10. Han X, Li Y, Xu Y, et al. Reversal of pancreatic desmoplasia by re-educating stellate cells with a tumour microenvironment-activated nanosystem. Nat Commun. 2018;9(1):3390. doi:10.1038/s41467-01805906-x

11. Lu J, Liu X, Liao YP, et al. Nano-enabled pancreas cancer immunotherapy using immunogenic cell death and reversing immunosuppression. Nat Commun. 2017;8(1):1811. doi:10.1038/ s41467-017-01651-9

12. Meng H, Nel AE. Use of nano engineered approaches to overcome the stromal barrier in pancreatic cancer. Adv Drug Deliv Rev. 2018;130:50-57. doi:10.1016/j.addr.2018.06.014

13. Akhter MH, Amin S. An investigative approach to treatment modalities for squamous cell carcinoma of skin. Curr Drug Deliv. 2017;14(5):597-612. doi:10.2174/1567201801666160906104254

14. Akhter MH, Rizwanullah M, Ahmad J, Ahsan MJ, Mujtaba MA, Amin S. Nanocarriers in advanced drug targeting: setting novel paradigm in cancer therapeutics. Artif Cells Nanomed Biotechnol. 2018;46(5):873-884. doi:10.1080/21691401.2017.1366333

15. Yang A, Lv Q, Chen F, Wang D, Liu Y, Shi W. Identification of recent trends in research on vitamin D: a quantitative and co-word analysis. Med Sci Monit. 2019;25:643-655. doi:10.12659/ MSM.913026

16. Wang L, Xue X, Zhang Y, Luo X. Exploring the emerging evolution trends of urban resilience research by scientometric analysis. Int J Environ Res Public Health. 2018;15(10):2181. doi:10.3390/ijerph15102181

17. Trujillo CM, Long TM. Document co-citation analysis to enhance transdisciplinary research. Sci Adv. 2018;4(1):e1701130. doi:10.1126/ sciadv. 1701130

18. Zou X, Yue WL, Vu HL. Visualization and analysis of mapping knowledge domain of road safety studies. Accid Anal Prev. 2018;118:131-145. doi:10.1016/j.aap.2018.06.010

19. Chen $\mathrm{C}, \mathrm{Hu} \mathrm{Z}$, Liu S, Tseng $\mathrm{H}$. Emerging trends in regenerative medicine: a scientometric analysis in CiteSpace. Expert Opin Biol Ther. 2012;12(5):593-608. doi:10.1517/14712598.2012.674507

20. Lu K, Yu S, Sun D, et al. Scientometric analysis of SIRT6 studies. Med Sci Monit. 2018;24:8357-8371. doi:10.12659/MSM.913644

21. Zhu X, Niu X, Li T, Liu C, Chen L, Tan G. Identification of research trends concerning application of stent implantation in the treatment of pancreatic diseases by quantitative and biclustering analysis: a bibliometric analysis. PeerJ. 2019;7:e7674. doi:10.7717/peerj.7674

22. Xiao F, Li C, Sun J, Zhang L. Knowledge domain and emerging trends in organic photovoltaic technology: a scientometric review based on CiteSpace analysis. Front Chem. 2017;5:67. doi:10.3389/ fchem.2017.00067

23. Li F, Li M, Guan P, Ma S, Cui L. Mapping publication trends and identifying hot spots of research on Internet health information seeking behavior: a quantitative and co-word biclustering analysis. $J \mathrm{Med}$ Internet Res. 2015;17(3):e81. doi:10.2196/jmir.3326 
24. Chen C. Searching for intellectual turning points: progressive knowledge domain visualization. Proc Natl Acad Sci U S A. 2004;101 (Suppl 1):5303-5310. doi:10.1073/pnas.0307513100

25. van Eck NJ, Waltman L. Software survey: VOSviewer, a computer program for bibliometric mapping. Scientometrics. 2010;84 (2):523-538. doi:10.1007/s11192-009-0146-3

26. van Eck NJ, Waltman L. Citation-based clustering of publications using CitNetExplorer and VOSviewer. Scientometrics. 2017;111 (2):1053-1070. doi:10.1007/s11192-017-2300-7

27. Elgogary A, Xu Q, Poore B, et al. Combination therapy with BPTES nanoparticles and metformin targets the metabolic heterogeneity of pancreatic cancer. Proc Natl Acad Sci U S A. 2016;113(36):E53285336. doi:10.1073/pnas.1611406113

28. Mattheolabakis G, Wang R, Rigas B, Mackenzie GG. Phospho-valproic acid inhibits pancreatic cancer growth in mice: enhanced efficacy by its formulation in poly-(L)-lactic acid-poly(ethylene glycol) nanoparticles. Int J Oncol. 2017;51(4):1035-1044. doi:10.3892/ijo.2017.4103

29. Barbero F, Russo L, Vitali M, et al. Formation of the protein corona: the interface between nanoparticles and the immune system. Semin Immunol. 2017;34:52-60. doi:10.1016/j.smim.2017.10.001

30. Chang X, Zhou X, Luo L, Yang C, Pan H, Zhang S. Hotspots in research on the measurement of medical students' clinical competence from 2012-2016 based on co-word analysis. BMC Med Educ. 2017;17(1):162. doi:10.1186/s12909-017-0999-8

31. Huang J, Tang J, Qu Y, et al. Mapping the knowledge structure of neonatal hypoxic-ischemic encephalopathy over the past decade: a co-word analysis based on keywords. J Child Neurol. 2016;31 (6):797-803. doi:10.1177/0883073815615673
32. Liu B, Chen H, Huang X. Map changes and theme evolution in work hours: a co-word analysis. Int J Environ Res Public Health. 2018;15 (5). doi:10.3390/ijerph15061188

33. Yang F, Jin C, Subedi S, et al. Emerging inorganic nanomaterials for pancreatic cancer diagnosis and treatment. Cancer Treat Rev. 2012;38 (6):566-579. doi:10.1016/j.ctrv.2012.02.003

34. Yang L, Mao H, Cao Z, et al. Molecular imaging of pancreatic cancer in an animal model using targeted multifunctional nanoparticles. Gastroenterology. 2009;136(5):1514-1525.e1512. doi:10.1053/j. gastro.2009.01.006

35. Kar S, Gajewicz A, Puzyn T, Roy K. Nano-quantitative structure-activity relationship modeling using easily computable and interpretable descriptors for uptake of magnetofluorescent engineered nanoparticles in pancreatic cancer cells. Toxicol in Vitro. 2014;28 (4):600-606. doi:10.1016/j.tiv.2013.12.018

36. David KI, Jaidev LR, Sethuraman S, Krishnan UM. Dual drug loaded chitosan nanoparticles-sugar-coated arsenal against pancreatic cancer. Colloids Surf B Biointerfaces. 2015;135:689-698. doi:10.1016/j. colsurfb.2015.08.038

37. Ahmad J, Ahmad MZ, Akhter H. Surface-engineered cancer nanomedicine: rational design and recent progress. Curr Pharm Des. 2020;26(11):1181-1190. doi:10.2174/1381612826666200214110645

38. Li Y, Hao L, Liu F, et al. Cell penetrating peptide-modified nanoparticles for tumor targeted imaging and synergistic effect of sonodynamic/HIFU therapy. Int $J$ Nanomedicine. 2019;14:5875-5894. doi:10.2147/IJN.S212184
International Journal of Nanomedicine

\section{Publish your work in this journal}

The International Journal of Nanomedicine is an international, peerreviewed journal focusing on the application of nanotechnology in diagnostics, therapeutics, and drug delivery systems throughout the biomedical field. This journal is indexed on PubMed Central, MedLine, CAS, SciSearch ${ }^{\mathbb{}}$, Current Contents ${ }^{\mathbb{R}} /$ Clinical Medicine, $^{2}$

\section{Dovepress}

Journal Citation Reports/Science Edition, EMBase, Scopus and the Elsevier Bibliographic databases. The manuscript management system is completely online and includes a very quick and fair peer-review system, which is all easy to use. Visit http://www.dovepress.com/ testimonials.php to read real quotes from published authors. 